\title{
BMJ Open Attitudes and perceptions of GPs and community pharmacists towards their role in the prevention of bisphosphonate-related osteonecrosis of the jaw: a qualitative study in the North East of England
}

\author{
Andrew Sturrock, ${ }^{1}$ Philip Preshaw, ${ }^{2}$ Catherine Hayes, ${ }^{1}$ Scott Wilkes ${ }^{1}$
}

To cite: Sturrock A, Preshaw $P$, Hayes C, et al. Attitudes and perceptions of GPs and community pharmacists towards their role in the prevention of bisphosphonate-related osteonecrosis of the jaw: a qualitative study in the North East of England. BMJ Open 2017;7:e016047. doi:10.1136/ bmjopen-2017-016047

- Prepublication history and additional material for this paper are available online. To view please visit the journal (http:// dx.doi.org/10.1136/bmjopen2017-016047).

Received 19 January 2017

Revised 6 July 2017

Accepted 7 July 2017

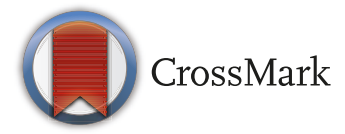

${ }^{1}$ Faculty of Health Sciences and Wellbeing, University of Sunderland, Sunderland, UK

${ }^{2}$ Centre for Oral Health Research and Institute of Cellular

Medicine, Newcastle University, Newcastle upon Tyne, Tyne and Wear, UK

Correspondence to Andrew Sturrock; andrew.sturrock@sunderland. ac.uk

\section{ABSTRACT}

Background Bisphosphonate-related osteonecrosis of the jaw (BRONJ) is a rare, yet significant, adverse effect of bisphosphonate therapy. A multidisciplinary approach to the prevention of BRONJ is recommended due to the significant morbidity and difficulty treating the condition. Current evidence suggests that both general practitioners (GPs) and community pharmacists have limited knowledge relating to BRONJ and that preventative strategies are rarely implemented.

Objective To explore the attitudes and perceptions of GPs and community pharmacists on the risks and preventative strategies for the development of BRONJ.

Design Interpretivist methodological approach using qualitative semistructured interviews.

Participants 9 community pharmacists and $8 \mathrm{GPs}$. Setting Primary Care in North East England and Cumbria, UK.

Methods Using a Grounded Theory methodology and integrating a process of constant comparison in the iterative enrichment of data sets, semistructured interviews were undertaken, transcribed and analysed using framework analysis. Salient themes were identified and related back to extant literature in the field.

Results Four salient and inter-related themes emerged: (1) uncertain knowledge, indicating limited exposure of respondents to BRONJ, and limited awareness of the implications of its diagnosis, risk factors and preventative strategies; (2) patient specific, referring to the complexity of patients, patient education and prioritising aspects of care; (3) wider context, indicating a lack of interdisciplinary communication and referral processes between professions, workload pressures, access and patient receptivity to dental services; and (4) professional, reflecting professional roles and responsibilities, authority and educational initiatives

Conclusions Effective communication or collaborative care between GPs and community pharmacists for the prevention of BRONJ is not apparent. Interventions to mitigate against the risk of developing BRONJ and clarity of GP and community pharmacy roles are required.
Strengths and limitations of this study

- Although bisphosphonate-related osteonecrosis of the jaw is not a common finding, affected patients experience significant morbidity, and management of this condition warrants further study to stimulate improved patient care.

- A qualitative approach yielded rich data through indepth semistructured interviews with two groups of healthcare professionals (general practitioners and community pharmacists). Constant comparison with concurrent data collection and analysis allowed further exploration and refining of emerging themes.

- A study limitation was that, although consistent with the methodological approach, the sample size was relatively small. Furthermore, the study was conducted in the North East of England and Cumbria, which may impact on transferability of the findings to other settings.

\section{INTRODUCTION}

Bisphosphonates are a class of drugs used in a variety of therapeutic indications, such as osteoporosis, Paget's disease, hypercalcaemia of malignancy, osteolytic bone metastases and osteolytic lesions of multiple myeloma. ${ }^{1}$ In practice, they are most commonly prescribed in the management of osteoporosis.

The prescribing of bisphosphonates has increased considerably over recent years. Statistical evaluation of prescribing in England reveals a rise of $122.6 \%$ in the number of individual prescription items dispensed between 2004 and 2014. ${ }^{2}$ Alendronic acid is the most commonly prescribed bisphosphonate, with 7391000 individual dispensations in $2014 .^{2}$ This rise may be attributable to increases in the proportion of elderly people in the UK population, publication of guidance 
recommending the prescribing of bisphosphonates and the availability of generic products. Risk for bisphosphonate-related osteonecrosis of the jaw (BRONJ) in people taking bisphosphonates is hypothesised to be related to the unique nature of the blood supply, structure and function of the jaw bones. ${ }^{3}$ Case reports of BRONJ emerged in the early 2000s; it is now well documented in the literature, ${ }^{4}$ and has been subject to a number of prescribing safety alerts in recent years. ${ }^{35}$

The actual incidence and prevalence rates of BRONJ are difficult to quantify, with varying reports in the literature. This is potentially attributable to a low incidence of reporting, the variance in diagnostic criteria and a percentage of mild self-resolving cases remaining undiagnosed. A nationwide study in the UK of patients presenting to departments of oral surgery, oral medicine, oral and maxillofacial surgery and dental hospitals identified 369 cases of BRONJ over a specified 2-year period. Oral bisphosphonates had been prescribed for $56 \%$ of the patients. Extrapolation of these data suggests that the incidence of BRONJ may be $8.2-12.8$ cases/million of the population/year, which is equivalent to 508-793 patients/year in the UK. ${ }^{6}$

Ideally, optimal dental health should be established before patients commence bisphosphonate therapy. ${ }^{7}$ This is to prioritise care that will subsequently reduce mucosal trauma or act prophylactically to aid in the avoidance of subsequent dental extractions or conditions which may further predispose the patient to oral surgery or dental procedures that impact on the osseous structures of the jaw. ${ }^{8}$

Several prospective studies have identified that dental screening and preventative strategies reduce the risk of osteonecrosis of the jaw. A study by Dimopoulous ${ }^{9}$ found a statistically significant reduction in the incidence of BRONJ with the implementation of preventative measures and Vandone et $a l^{10}$ reported a $50 \%$ reduction in the incidence rate with screening and pretreatment preventative dental care. ${ }^{9}{ }^{10}$ A multidisciplinary approach to the prevention of BRONJ is recommended in the literature for the management of patients requiring bisphosphonate therapy, ${ }^{11} 12$ incorporating both patient and health professional education of the risk of the development of BRONJ. ${ }^{5}$ Education of dentists, pharmacists, general practitioners (GPs) and patients about BRONJ is indicated, ${ }^{13}$ with specific emphasis on the provision of focused preventative measures and detailed oral hygiene instructions. ${ }^{14}$

Available published evidence describing the attitudes of both GPs and pharmacists towards, and their perceptions of, their roles in preventive strategies for BRONJ is limited. A questionnaire survey of GPs $(n=120)$ and pharmacists $(n=60)$ in North Wales identified that although both sets of healthcare professionals have regular contact with patients who are prescribed bisphosphonates, they have limited knowledge of the dental implications associated with treatment. Both groups of professionals reported awareness of the side effects of bisphosphonates; however, only $11.8 \%$ of GPs and $9.7 \%$ of pharmacists specifically identified osteonecrosis as a potential unwanted effect of therapy. ${ }^{15}$

Furthermore, even when pharmacists and GPs report some knowledge of BRONJ, is it not clear how this awareness influences their clinical practice. The aim of this study was to explore the attitudes and perceptions of GPs and community pharmacists on the risks and preventative strategies for the development of BRONJ.

\section{METHOD}

\section{Design}

A Grounded Theory approach ${ }^{16}$ with constant comparison was used throughout the research. Semistructured, one-to-one interviews were carried out by a single researcher (AS), at either the School of Pharmacy or the participant's workplace, depending on participant preference and availability. The interviews were audio-recorded and transcribed verbatim; field notes were not taken due to verbatim transcribing. Integrating a process of constant comparison, ${ }^{17}$ an initial topic guide (see online supplementary 1) was produced and refined by the research team; this served as a benchmark of questioning, which was subsequently developed iteratively as data were progressively enriched.

\section{Setting}

Participants were recruited from a range of urban and rural primary care locations in the North East of England and Cumbria. GPs were recruited from both teaching and non-teaching practices and community pharmacists were recruited from independent (single or small-chain pharmacies) and multiple pharmacies (companies consisting of numerous pharmacy stores) (table 1).

\section{Participants}

Seventeen participants, nine community pharmacists and eight GPs were recruited to the study. An invitation letter and participant information sheet (see online supplementary file 2) were posted to GPs and community pharmacists. An initial convenience sample of participants who responded to the invitation was implemented with further recruitment achieved via snowball sampling. No participants who responded to the invitation refused to participate or dropped out of the study.

\section{Analysis}

Constant comparison allowed enrichment of data and for new concepts to guide subsequent interviews via the strategic development of each subsequent topic guide. Adoption of Ritchie and Spencer's framework analysis ${ }^{18}$ allowed salient themes from the findings to be identified. Data were analysed by AS, using Microsoft Word 2010 and Microsoft Excel 2010, with transcripts and emerging themes cross-checked for interpretation and agreed among the research team until saturation occurred; transcripts were not returned to participants for comment 
Table 1 Participant characteristics-pharmacists

\begin{tabular}{lllllll}
\hline Participant & Gender & $\begin{array}{l}\text { Number of years } \\
\text { since registration }\end{array}$ & $\begin{array}{l}\text { Number of items } \\
\text { dispensed per month }\end{array}$ & $\begin{array}{l}\text { Practice } \\
\text { location }\end{array}$ & Independent/multiple & Full/part-time \\
\hline P1 & Female & $6-10$ & $6000-8999$ & Suburban & Independent & Full-time \\
P2 & Female & $11-15$ & $12000+$ & Suburban & Multiple & Full-time \\
P3 & Female & $0-5$ & $3000-5999$ & Suburban & Multiple & Full-time \\
P4 & Female & $21+$ & $3000-5999$ & Urban & Independent & Part-time \\
P5 & Female & $0-5$ & $6000-8999$ & Urban & Independent & Full-time \\
P6 & Female & $0-5$ & $6000-8999$ & Urban & Independent & Full-time \\
P7 & Male & $6-10$ & $6000-8999$ & Urban & Multiple & Full-time \\
P8 & Female & $16-20$ & $6000-8999$ & Rural & Independent & Part-time \\
P9 & Male & $11-15$ & $6000-8999$ & Semirural & Multiple & Full-time \\
\hline P. & & & & &
\end{tabular}

P, pharmacist.

or feedback and repeat interviews were not performed. A sample transcript has been published alongside this paper (see online supplementary file 3). Framework analysis involved a five-stage process: (1) familiarisation with the data-interviews were transcribed by AS and key issues identified through immersion in the data; achieved via iterative cycles of reading and rereading of transcripts; (2) development of a thematic framework-the initial themes formed the basis of a thematic framework; (3) indexing data-data were indexed against the thematic framework; (4) charting-charts were produced of the data within the thematic framework; (5) mapping of the data-themes were reviewed until definitive concepts could be produced from the data.

\section{Ethics}

Ethical approval was obtained from the University of Sunderland (Reference PHW52).

\section{RESULTS}

Seventeen healthcare professionals were included in this study (tables 1 and 2). Interviews were carried out between January and October 2016; 1 hour was designated for each interview.
Four salient inter-related themes emerged: (1) uncertain knowledge - a lack of familiarity with the subject area, the prevalence and significance of BRONJ and limited exposure to the condition; (2) patient specificcomplexity of patients, clinical priorities and patient education; (3) wider context-access/fear of dental services, interprofessional communication and clinical workload; (4) professional-perceived responsibilities, authority and interprofessional education.

\section{Uncertain knowledge}

All participants perceived themselves to have some degree of knowledge on the adverse effects that are associated with bisphosphonate therapy. The concept of BRONJ was introduced in the participant information sheet and opened up for discussion during the interview; participants actually had minimal knowledge on this topic but all were aware of the potential risk.

'I think it was probably sitting way at the back of my mind...it was probably in a lecture at some point.' (GP4)

Although poor dental health and the duration of therapy were frequently identified, all of the participants

\begin{tabular}{|c|c|c|c|c|c|c|}
\hline Participant & Gender & $\begin{array}{l}\text { Number of years } \\
\text { since registration }\end{array}$ & $\begin{array}{l}\text { Practice size } \\
\text { (patients) }\end{array}$ & Practice location & $\begin{array}{l}\text { Teaching } \\
\text { practice }\end{array}$ & Full/part-time \\
\hline GP2 & Male & $16-20$ & 12 000+ & Urban & Teaching & Part-time \\
\hline GP3 & Male & $21+$ & $12000+$ & Suburban & Teaching & Part-time \\
\hline GP6 & Female & $16-20$ & $3000-5999$ & Rural & Teaching & Part-time \\
\hline GP7 & Male & $21+$ & $9000-11999$ & Semirural & Teaching & Full-time \\
\hline GP8 & Male & $16-20$ & $9000-11999$ & Semirural & Teaching & Full-time \\
\hline
\end{tabular}

GP, general practitioner. 
had limited awareness of the risk factors for the development of BRONJ.

'I am not aware of any, I imagine that significant dental problems would be associated with it, but I am not actually aware of any others.' (GP3)

Participants were uncertain on the prevalence of BRONJ and had limited knowledge on the significant morbidity associated with the condition.

'I have never seen it, so I presume it's not very common...I don't really know how serious it is when it does happen.' (P3)

One GP had first-hand experience of managing patients with BRONJ, and the significant morbidity that her patients had experienced influenced their attitude towards management of patients who are prescribed bisphosphonates. None of the other participants had been involved with the care of a patient with BRONJ.

'It's the sort of thing that once you see it, you then remember it. They were both very complex patients, but the amount of morbidity involved with the osteonecrosis of the jaw in both of those patients was considerable.' (GP1)

\section{Patient specific}

Patients prescribed bisphosphonates usually have a number of comorbidities. They are often elderly and are prescribed multiple medications, and their management can be complex. Indeed, this complexity requires that practitioners assign priorities in their care, relating to both the overall management of the patient and to more specific priorities related to bisphosphonates.

'They are lower down in the pecking order of things that we look at when we are supervising polypharmacy, when we are looking at chronic disease management.' (GP3)

All participants identified bisphosphonates as having very specific administration instructions and common side effects, such as gastrointestinal (GI) or oesophageal problems; these were the focus of consultations. However, participants were concerned about overloading patients with information and the risk of patients potentially refusing treatment.

'You try not to overload them with too much information because you know that sometimes they can't even take it on board at the best of times.' (P2)

Patient education was a key issue that emerged from the data; participants placed importance specifically on the education of patients in relation to administration instructions and common side effects of bisphosphonates. This would usually take the form of a set of predefined counselling points.
'I think when you have a drug like a bisphosphonate, which is complex with its instruction on how to take it and people are tied up in that.' (GP1)

Although some participants advised bisphosphonate patients to seek dental check-ups, most reported that many of their patients, in general, appeared to not appreciate the importance of achieving and maintaining good dental health through self-performed daily oral hygiene and regular dental check-ups. This was a common theme reported by participants in relation to patients' outlook on oral health issues as a whole and not just related to the specific preventative strategies for BRONJ. This was identified as a barrier in the management of this patient population and a focus for patient education.

'I would say that their oral hygiene was not particularly great. I think it's probably just not wanting to go to the dentist and fear of the dentist.' (P9)

Patients often tend to forget the initial advice given to them and reminders or continuous advices are necessary to enhance patient education. Teamwork highlights the importance of specific counselling and reinforces the advice that is given to patients.

'If a new drug is initiated, that is the time to reinforce what the patients been told about the drug and you know to give them the message. I think the more reinforcement and the more information the better.' (GP2)

\section{Wider context}

Both GPs and pharmacists identified that there is reluctance among certain patients to seek dental advice. A number of reasons were proposed for this, including the cost of dental treatment, a general lack of oral health awareness and patients with dental phobias.

'The processes of how you get people to take their dental health seriously are very difficult. The ones that pay for dentistry are likely to be the ones with good teeth, the others who get free treatment just don't access it.' (GP3)

Access to dentists was also felt to be an issue that both pharmacists and GPs had encountered, specifically the availability of dental services for patients and referral pathways between professions.

'Some people don't even have an NHS dentist. I am aware of where I work, there was a dentist upstairs, but it wasn't an NHS dentist. I think when you want to refer someone to another service you know it is going to be a little bit more problematic than just making an appointment with a GP for example.' (P2)

Participants all described a heavy workload and that in the small amount of time that they had with each patient, they would have to prioritise the information they gave to patients. 
'In that 2 min that you have got to hand something out to somebody, you concentrate on the important things, such as how to take it, to get their concordance and compliance.' (P2)

A lack of communication between both GPs/pharmacists and dentists was identified as a major barrier. The absence of a formal referral process between pharmacists, GPs and the dental profession was highlighted throughout. This was felt to be an issue related to BRONJ and represented a wider problem in the management of oral health in primary care. In order to successfully manage the risk of BRONJ, it was clear from interviewees that communication between professionals is key.

'I think maybe there needs to be a little bit more communication involved with pharmacists. The triangle, pharmacist, dentist and prescriber.' (P2)

'Some sort of shared record keeping where you could enter into the system. You have done a review and these side effect were discussed with the patient, that would be brilliant. That would make it part of that clinical record, I'd know about it, the patient would know about it. I think that would work very well.' (GP7)

One of the key areas identified by all pharmacists and some of the GPs was the benefit of Medication Use Reviews (MURs) and the New Medicine Service (NMS) in community pharmacies. The MUR and NMS services are both advanced service within the National Health Service (NHS) Community Pharmacy Contractual Framework in England. An MUR is a structured, adherence-centred review of patients prescribed multiple medicines and the NMS service provides support for patients with longterm conditions that have been newly prescribed a medicine. $^{1920}$

These services provide pharmacies with both the time and structure to provide more detailed advice to patients on medications. Bisphosphonates are not currently specified in either service. Although it was felt that many drugs should be included, all participants identified that bisphosphonates should be included in these services due to their specific administration instructions and potential for side effects.

'I think during an MUR you certainly have more time to focus on the individual drugs and then it kind of triggers in your brain the more important things that you should be speaking to them about.' (P2)

\section{Professional}

GPs acknowledged their role as the prescriber and the need to counsel patients on the side effects of their medication. Both prescribers and pharmacists were in agreement that pharmacists are the experts on medications and they have a role to play in counselling patients on safe and effective use of medicines.
'I think counselling about medication is far better done by the pharmacists. I think the other reason is perhaps, when a patient sees a doctor they expect to be able to discuss all aspects of their lives and their care. When they see the pharmacist, they know they are seeing the pharmacist about their medication. I think it is much easier for the pharmacist to keep the patient focused on the drugs and the patient to stay focused on the drugs.' (GP1)

Although pharmacists acknowledged their role in counselling patients on medications, a number of them felt that if a patient needs to be dentally fit before commencing bisphosphonate therapy, then it would be the responsibility of the GP to arrange this. Although in many cases GPs would be responsible for initially prescribing bisphosphonates and their continued prescribing, it was commented that bisphosphonates can, at times, be initiated in secondary care. This was certainly the case for intravenous bisphosphonates with all GPs and pharmacists reporting little or no experience with prescribing or dispensing these products. As intravenous bisphosphonates are usually prescribed in secondary care, it was felt by some of the participants that this was a potential risk, as they can be missed on medication lists.

'Making sure that the dental check has been done and that they're healthy should actually be done before you prescribe medication, because if you prescribe a medicine without knowing that, then technically how do you know that it's going to be safe for the patient to take. I think my role as a pharmacist is certainly to promote that it's been done, and if it hasn't to take further steps with the patient.' (P7)

A number of participants also described limited education or training in relation to oral and dental health.

'We have no training in dental care. You know to brush your teeth and that's what you say to people. I think, I don't know, maybe we should have some more training.' (GP5)

'No not really, a little bit maybe in lectures at university but not with dentists, we have worked quite closely with the doctors but not with dentists.' (P1)

\section{DISCUSSION}

\section{Summary of main findings}

It is apparent that both sets of participants (GPs and pharmacists) had limited knowledge of BRONJ, in particular in relation to its prevalence and the morbidity associated with the condition. As BRONJ is relatively uncommon, the majority of participants also lacked first-hand experience of managing affected patients.

Due to the complexity of this patient group and bisphosphonates as a therapeutic class, interviewees assigned priorities in relation to clinical management and in patient education. Consultations would usually focus 
on the specific administration requirements and more common GI-related adverse effects as opposed to the risk of developing BRONJ and the need for good oral and dental health.

Awareness of the issue was thought to be a key barrier to implementing preventative strategies in this patient group; however, wider issues in relation to the attitudes of patients towards oral health, a reluctance to attend the dentist and difficulties in accessing dental services were thought to be potential barriers for patients. The lack of communication between the professions was also cited as a key issue that needs to be addressed for the successful implementation of any future collaborative preventative strategies in this patient group, with the MUR and NMS pharmacy services identified as a potential facilitator.

Pharmacists and GPs reported good working relationships but interprofessional educational opportunities with dental colleagues appear to have been limited in scope or non-existent, and were cited as a potential enabler for improving multidisciplinary working.

\section{Comparison with existing literature}

Knowledge on the oral risks associated with bisphosphonate therapy has been reported to be limited. ${ }^{15}$ All participants interviewed in this research reported being aware of the risk, although this was introduced before the interviews in the participant information leaflet.

Many of the participants would not routinely mention the risk of osteonecrosis of the jaw when prescribing bisphosphonates or when counselling patients about the medication. This is consistent with a small quantitative study that identified only $17 \%$ of patients prescribed oral bisphosphonates were aware of the risk of BRONJ, with the majority of these patients acquiring this knowledge from patient information leaflets and not from their GP. ${ }^{21}$

All participants reported reluctance among patients to attend dental appointments, with a significant proportion of their patients being either not registered with a dentist or not regular attendees. This is consistent with NHS dental statistics, which state that only $52 \%$ of the adult population has seen an NHS dentist within the previous 24 months. ${ }^{22}$

A number of clinical guidelines and patient safety alerts recommend that patients should be counselled on the risk of BRONJ and advised to seek a dental check-up prior to initiating bisphosphonate therapy. ${ }^{58}$ Our data suggest that this does not appear to routinely happen. A recent study in Japan reported that $62 \% \quad(n=629)$ of physicians did not request oral healthcare by a dentist before commencing bisphosphonate therapy and $72 \%$ of participants reported no cooperation between physicians and dentists. They concluded that a strategy for sharing information among physicians, dentists and patients is required to reduce the incidence of osteonecrosis of the jaw associated with osteoporosis treatment. ${ }^{23}$ The population studied were all members of the Japan Osteoporosis Society; the nature of this sample and therefore interest in osteoporosis management of the participants could potentially explain the higher rates of dental referrals than reported in other studies.

The MUR and NMS were identified as potential facilitators in the prevention of BRONJ. Bisphosphonates are not directly specified in either of these services at present, although participants were in agreement that it would be beneficial for them to be included. The literature to support both services is mixed; a detailed review by the University of Nottingham found that the implementation of the NMS was constrained by the quality of the pharmacist's relationship with GPs. They found that poor communication between the professions and a lack of awareness or understanding by GPs about the service resulted in a lack of referrals; this is consistent with statements from some of the GPs in this study. Pharmacists also suggested that GPs were not interested in the NMS as it potentially encroached on professional boundaries and duplicated work undertaken by the GP. ${ }^{24}$ In comparison, the GPs in this study, despite having limited knowledge of the service, were all supportive of its role and the reinforcement of important counselling points was thought to be a key responsibility of the pharmacist.

Pharmacists are subject to organisational pressures to meet targets around the MUR service which has been reported to result in their offering the service to patients who meet the minimum inclusion criteria and avoiding offering the service to more complex patients due to time pressures. ${ }^{25}$ This potentially impacts the patient group under study as a clear theme that emerged from the data was the complexity and polypharmacy issues of patients taking bisphosphonates.

An ethnographic study utilising observations and patient interviews in two English community pharmacies found that patients generally were positive about the MUR, and patients tended to view the pharmacist as an expert on medicines. However, some participants felt wary of the pharmacist's involvement, considering that the pharmacists were deliberately or intentionally bypassing the GP. This study also found that there was little evidence to suggest that the professions were collaborating to identify patients who could benefit from the service. ${ }^{25}$

\section{Limitations}

The study was based around the a priori issue of limited knowledge among GPs and pharmacists in the prevention of BRONJ; the concept of BRONJ was introduced during the patient information leaflet, therefore exposing participants to the concept before the interview.

Participants were all located in the North East of England and Cumbria; this therefore may impact on the transferability of findings to other geographical locations or healthcare settings. For example, a variation in the access to dental services in a particular location may influence the practice of participants and patients.

\section{Future work and implications for clinical practice}

This study has highlighted a number of areas for future study. However, missing from this study and the 
wider literature is the dental profession's insight into the interprofessional prevention of BRONJ. A recent publication in British Dental Journal highlighted the opportunities for interprofessional working between pharmacists and dentists; with a particular focus on chronic diseases, it was suggested that dental and pharmacy teams should take action to improve communication and devise schemes for collaborative working. ${ }^{26}$ Published clinical guidelines recommend that patients should be referred for dental assessment and treatment prior to initiation on bisphosphonate therapy, but it is apparent this is not happening. The impact of this on dentists and their perspective on how the professions can collaborate to improve patient care would be important to consider before implementing any preventative strategies.

Raising awareness of the rare side effects of medicines is an important consideration when prescribing; explicitly pointing out rare side effects may create adherence problems and result in non-compliance with a potentially beneficial medicine which needs to be balanced against fully informing patients about the associated risks. Further research with patients to explore this issue would help guide practitioners and would be applicable to many other rare conditions and medicines.

The patient remains the central focus of the healthcare team, and therefore engaging patients in the management of their health is essential when introducing prevention strategies for BRONJ. Attitudes of patients towards the roles of the various team members and their priorities or expectations when being prescribed a new medicine will guide the development of such services.

\section{CONCLUSION}

Both GPs and pharmacists demonstrated relatively limited knowledge in relation to BRONJ and the preventative strategies recommended in the literature. Patients prescribed bisphosphonates often have complex medical histories, requiring practitioners to assign priorities in their management and, as such, the measures required to prevent the development of BRONJ can be overlooked.

Prescribing rates of bisphosphonates are increasing, with an ageing population and increasing emphasis on treating and preventing conditions such as osteoporosis. Therefore, the incidence of BRONJ is likely to increase; this may continue to be the case unless changes are made to current practice. Preventive measures should be implemented and further research performed to assess the effectiveness of such interventions.

\section{Acknowledgements We thank the participants who generously gave their time.}

Contributors An interest in the subject area was developed by AS as an extension to the interprofessional learning opportunities delivered at the University of Sunderland School of Pharmacy. The multidisciplinary team was assembled to reduce bias and provide rigour in the investigation. AS and SW designed the study. AS recruited the participants and carried out the study. AS identified the thematic framework and interpreted the data. AS, SW, PP and $\mathrm{CH}$ reviewed and refined the data. AS wrote the paper and all authors revised it. AS received training in qualitative research skills by the research team and through attendance at a Qualitative Research Methods in Health Course at University College London.

Competing interests None declared.

Ethics approval University of Sunderland Research Ethics Committee.

Provenance and peer review Not commissioned; externally peer reviewed.

Data sharing statement No additional data are available.

Open Access This is an Open Access article distributed in accordance with the Creative Commons Attribution Non Commercial (CC BY-NC 4.0) license, which permits others to distribute, remix, adapt, build upon this work non-commercially, and license their derivative works on different terms, provided the original work is properly cited and the use is non-commercial. See: http://creativecommons.org/ licenses/by-nc/4.0/

(c) Article author(s) (or their employer(s) unless otherwise stated in the text of the article) 2017. All rights reserved. No commercial use is permitted unless otherwise expressly granted.

\section{REFERENCES}

1. Fliefel R, Tröltzsch M, Kühnisch J, et al. Treatment strategies and outcomes of bisphosphonate-related osteonecrosis of the jaw (BRONJ) with characterization of patients: a systematic review. Int $J$ Oral Maxillofac Surg 2015;44:568-85.

2. Health and Scocial Care Information Centre. Prescriptions dispensed in the community, England 2004-2014, Leeds, Health and Social Care Information Centre 2015. http://www.hscic.gov.uk/catalogue/ PUB17644/pres-disp-com-eng-2004-14-rep.pdf (accessed Apr 05 16).

3. Medicines Healthcare Regulatory Agency. Drug Safety Update: Bisphosphonates: osteonecrosis of the jaw 2009 https://www.gov. uk/drug-safety-update/bisphosphonates-osteonecrosis-of-the-jaw (accessed Nov 15 2015).

4. McLeod NM, Brennan PA, Ruggiero SL. Bisphosphonate osteonecrosis of the jaw: a historical and contemporary review. Surgeon 2012;10:36-42.

5. European Medicines Agency. CHMP assessment report on bisphosphonates and osteonecrosis of the Jaw. London: European Medicines Agency, 2009. http://www.ema.europa.eu/docs/en_GB/ document_library/Report/2010/01/WC500051428.pdf. (accessed Sep 02 2016).

6. Rogers SN, Palmer NO, Lowe D, et al. United Kingdom nationwide study of avascular necrosis of the jaws including bisphosphonaterelated necrosis. Br J Oral Maxillofac Surg 2015;53:176-82.

7. Otomo-Corgel J. Osteoporosis and osteopenia: implications for periodontal and implant therapy. Periodontol 2000 2012;59:111-39.

8. Scottish Dental Clinical Effectiness Programme. Oral Health Management of Patients Prescribed Bisphosphonates: Dental Clinical Guideline. Dundee: Scottish Dental Clinical Effectiness Programme, 2011. http://www.sdcep.org.uk/published-guidance/ bisphosphonates. (accessed Apr 05 2016).

9 Dimopoulos MA, Kastritis E, Bamia C, et al. Reduction of osteonecrosis of the jaw (ONJ) after implementation of preventive measures in patients with multiple myeloma treated with zoledronic acid. Ann Oncol 2009;20:117-20.

10 Vandone AM, Donadio M, Mozzati M, et al. Impact of dental care in the prevention of bisphosphonate-associated osteonecrosis of the jaw: a single-center clinical experience. Ann Oncol 2012;23:193-200.

11. Shannon J, Shannon J, Modelevsky S, et al. Bisphosphonates and osteonecrosis of the jaw. J Am Geriatr Soc 2011;59:2350-5.

12. Patel V, McLeod NM, Rogers SN, et al. Bisphosphonate osteonecrosis of the jaw-a literature review of UK policies versus international policies on bisphosphonates, risk factors and prevention. Br J Oral Maxillofac Surg 2011;49:251-7.

13. Rayman S, Almas K, Dincer E. Bisphosphonate-related jaw necrosis: a team approach management and prevention. Int J Dent Hyg 2009;7:90-5.

14. Gaudin E, Seidel L, Bacevic M, et al. Occurrence and risk indicators of medication related osteonecrosis of the jaw after dental examination: a systematic review and meta-analysis. $J$ Clin Periodontol 2015;45:922-32.

15. Masson D, O'Callaghan E, Seager M. The knowledge and attitudes of North Wales healthcare professionals to bisphosphonate associated osteochemonecrosis of the jaws. J Disab Oral Health 2009;10:175-83. 
16. Glaser BG, Strauss AL. The Discovery of Grounded Theory: Strategies for Qualitative Research. Chicago: Aldine, 1967.

17. Boeije H. A purposeful approach to the constant comparative method in the analysis of qualitative interviews. Quality and Quantity 2002;36:391-409.

18. Ritchie J, Spencer L. Qualitative data analysis for applied policy research. In: Humberman M, Miles M, eds. The qualitative researcher's companion. Thousand Oaks: Sage, 2002:305-29.

19. Pharmaceutical Services Negotiating Committee. Medicines Use Review. http://psnc.org.uk/services-commissioning/advancedservices/murs/ (accessed May 05 2017).

20. Pharmaceutical Services Negotiating Committee. New Medicines Service. http://psnc.org.uk/services-commissioning/advancedservices/nms/ (accessed May 05 2017).

21. Bauer JS, Beck N, Kiefer J, et al. Awareness and education of patients receiving bisphosphonates. J Craniomaxillofac Surg 2012;40:277-82.
22. Health and Social Care Information Centre. NHS Dental Statistics for England: 2015-16, Third Quarterly Report. Leeds, Health and Social Care Information Centre. 2016 http://www.hscic.gov.uk/catalogue/ PUB20837/nhs-dnet-stat-eng-15-16-q3-rep.pdf (accessed Jun 21 2016).

23. Taguchi A, Shiraki M, Sugimoto T, et al. Lack of cooperation between physicians and dentists during osteoporosis treatment may increase fractures and osteonecrosis of the jaw. Curr Med Res Opin 2016;32:1261-8.

24. Latif A, Waring J, Watmough D, et al. Examination of England's New Medicine Service (NMS) of complex health care interventions in community pharmacy. Res Social Adm Pharm 2016;12.

25. Latif $A$, Pollock K, Boardman HF. Medicines use reviews: a potential resource or lost opportunity for general practice? BMC Fam Pract 2013;14:57.

26. Wilson N, Soni A. Interprofessional working: a spearhead opportunity for dentistry and pharmacy. Br Dent J 2016;221:607-8. 
Correction: Attitudes and perceptions of GPs and community pharmacists towards their role in the prevention of bisphosphonate-related osteonecrosis of the jaw: a qualitative study in the North East of England

Sturrock A, Preshaw P, Hayes C, et al. Attitudes and perceptions of GPs and community pharmacists towards their role in the prevention of bisphosphonate-related osteonecrosis of the jaw: a qualitative study in the North East of England. BMJ Open 2017;7:e016047. doi: 10.1136/bmjopen-2017-016047.

The middle initial of author 'Philip Preshaw' is missing from the article. This name should be written 'Philip M Preshaw'.

Open Access This is an Open Access article distributed in accordance with the Creative Commons Attribution Non Commercial (CC BY-NC 4.0) license, which permits others to distribute, remix, adapt, build upon this work non-commercially, and license their derivative works on different terms, provided the original work is properly cited and the use is non-commercial. See: http://creativecommons.org/licenses/by-nc/4.0/

(C) Article author(s) (or their employer(s) unless otherwise stated in the text of the article) 2017. All rights reserved. No commercial use is permitted unless otherwise expressly granted.

BMJ Open 2017;0:e016047corr1. doi:10.1136/bmjopen-2017-016047corr1

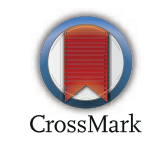

